\title{
Condensation driven by a quantum phase transition
}

\author{
Miguel Alvarer* and Jose Reslent \\ Coordinación de Física, Universidad del Atlántico, Carrera 30 Número 8-49, Puerto Colombia.
}

\begin{abstract}
The grand canonical thermodynamics of a bosonic system is studied in order to identify the footprint of its own high-density quantum phase transition. The phases displayed by the system at zero temperature establish recognizable patterns at finite temperature that emerged in the proximity of the boundary of the equilibrium diagram. The gaped phase induces a state of collectivism/condensation at finite temperature in which population cumulates into the ground state in spite of interacting attractively. The work sets the foundation to approach the effect of attraction in the formation of a molecular condensate.
\end{abstract}

Keywords: Phase Transitions, Boson Systems, Condensation.

\section{INTRODUCTION}

The understanding of collectivism is a crucial task in the search for practical applications where a number of phases taking place in the low-energy range could be utilized in mass-produced technology. The process by which a large number of particles, in particular bosons, collectively occupy the same single-body state gives rise to coherent phases where the features of a single state magnify and therefore manifest at the macroscopic level. The archetype of collectivism is Bose-Einstein condensation [1], a mechanism that constitutes the interpretative ground of other phenomena like superfluidity or superconductivity. In essence, condensation is driven by statistical effects at the single-body level, but interaction cannot be completely suppressed in any practical scenario. The role of attractive interaction, in particular, has proved detrimental in a number of studies [2 12], but experimental evidence of condensation in attractive systems has been reported [13]. In this context there must be a range over which collectivism can be sustained under the effect of interaction, especially when the interaction role can be captured using single-body terms. The relevance of this phenomenon is notorious in times when the first prototypes of quantum computation have demonstrated quantum supremacy [14, 15] and control mechanisms are increasingly necessary to extent the current capabilities. This has boosted the interest in the realization of novel collective phases such as the molecular condensate [16, 17], which in turn evidences the need of a better understanding of the process of condensation under attractive fields since it may prove a precursor of molecular formation.

Let us consider a system of one-species bosons that can tunnel between two equal-energy wells [18]. Bosons can interact among them only when they occupy the same well. This interaction is attractive, so it tends to pack bosons together. The system is modeled via the next quantized Hamiltonian

$$
\hat{H}_{M}=\delta\left(\hat{a}_{1}^{\dagger} \hat{a}_{2}+\hat{a}_{2}^{\dagger} \hat{a}_{1}\right)-i \gamma\left(\hat{a}_{1}^{\dagger} \hat{a}_{2}-\hat{a}_{2}^{\dagger} \hat{a}_{1}\right)-\frac{\lambda}{M}\left(\hat{m}_{1}^{2}+\hat{m}_{2}^{2}\right) .
$$

\footnotetext{
*Electronic address: migue leo93@hotmail.com
}

†Electronic address: reslenjo@yahoo.com
Ladder operators obey standard bosonic commutation rules, $\left[\hat{a}_{1}, \hat{a}_{1}^{\dagger}\right]=\left[\hat{a}_{2}, \hat{a}_{2}^{\dagger}\right]=1,\left[\hat{a}_{1}, \hat{a}_{2}\right]=0$. The number operators

$$
\hat{m}_{1}=\hat{a}_{1}^{\dagger} \hat{a}_{1}, \hat{m}_{2}=\hat{a}_{2}^{\dagger} \hat{a}_{2},
$$

determine the particle occupation at each well. Integer $M$ is the total number of particles in the system, so that $M=\hat{m}_{1}+\hat{m}_{2}$. Constants $\gamma$ and $\lambda$ modulate the intensity of hopping and interaction respectively. Both are considered strictly positive in this work. Constant $\delta$ modulates the intensity of a hopping process in which the phase of one well with respect to the other is unchanged. Henceforth the energy-scale is chosen so that $\delta=1$. By definition $\hat{H}_{0}=0$. Hamiltonian (11) has the essential elements of a Bose-Hubbard model, which has been studied from the perspective of the grand-canonical formalism as a descriptor of the transition between Mott insulator and superfluid 19 and also verified experimentally with ultracold atoms [20, 21]. The main difference with the current study is that here the interaction is attractive and has been scaled with respect to the number of particles. The latter feature can also be seen in related models such as the Lipkin-Meshkov-Glick model and the infinite-range Ising model, from which Hamiltonian (1) can be obtained as a second quantization [22].

Assuming that to first order the ground state adopts a collective form, it can be written as

$$
|G(\theta, \varphi)\rangle=\frac{\hat{b}_{1}^{\dagger}|0,0\rangle}{\sqrt{M !}}, \hat{b}_{1}^{\dagger}=\hat{a}_{1}^{\dagger} \cos \theta-\hat{a}_{2}^{\dagger} e^{i \varphi} \sin \theta,
$$

so that $\left[\hat{b}_{1}, \hat{b}_{1}^{\dagger}\right]=1$. The angle domains are $0 \leq \theta \leq$ $\pi / 2$ and $0 \leq \varphi<2 \pi$, ensuring that different angle pairs correspond to genuinely different modes. In order to find the correct angles, the energy function

$$
E(\theta, \varphi)=\left\langle G(\theta, \varphi) \mid \hat{H}_{M} G(\theta, \varphi)\right\rangle
$$

is minimized over $\theta$ and $\varphi$. From the procedure shown in appendix $\mathrm{A}$ it follows that the system undergoes two distinct phases determined by the value of $\lambda$ relative to the critical value $\lambda_{c}=\sqrt{1+\gamma^{2}}$. In the region $\lambda \leq \lambda_{c}$ the energy displays a single minimum at

$$
\theta^{\star}=\frac{\pi}{4}, \quad \cos \varphi^{\star}=\frac{1}{\lambda_{c}}, \quad \sin \varphi^{\star}=\frac{\gamma}{\lambda_{c}} .
$$


The corresponding ground state energy is

$$
E_{\lambda \leq \lambda_{c}}^{\star}=-M\left(\lambda_{c}+\frac{\lambda}{2}\right) .
$$

From quantum theory it is known that because in this phase the ground state is non-degenerate it must display the Hamiltonian's symmetries. Hamiltonian (11) commutes with the antiunitary operator composed by the swapping of subscripts $1 \leftrightarrow 2$ followed by complex conjugation. As a consequence, the number of particles on each well must be the same.

In the region $\lambda \geq \lambda_{c}$ the energy displays two equal minima located at $\left(\theta_{1}^{\star}, \varphi^{\star}\right)$ and $\left(\theta_{2}^{\star}, \varphi^{\star}\right)$. Here $\varphi^{\star}$ is the same than in equation (5). In addition

$$
\theta_{1}^{\star}=\frac{1}{2} \arcsin \frac{\lambda_{c}}{\lambda}, \theta_{2}^{\star}=\frac{\pi}{2}-\theta_{1}^{\star} .
$$

Notice that by making $\theta$ different from $\pi / 4$ the balance of occupation between the wells is broken and the proposed solutions do not independently display the Hamiltonian's inversion symmetry. The corresponding ground state energy is

$$
E_{\lambda \geq \lambda_{c}}^{\star}=-M\left(\lambda+\frac{\lambda_{c}^{2}}{2 \lambda}\right) .
$$

The phase change is rooted in a change in the Hamiltonian spectrum, which goes from non-degenerate for $\lambda<\lambda_{c}$ to two-fold degenerate for $\lambda \geq \lambda_{c}$. In the latter case the equilibrium configuration corresponds to a maximally mixed state in the space spanned by two linearly independent ground states. However, it is often argued that small perturbations tip the scale to either of the constituent pure-states, such that the phase change is equivalent to a continuous break of symmetry of a pure ground-state as a function of the Hamiltonian's parameters. This process is known as a quantum phase transition and takes place at zero temperature since it is in this instance that the equilibrium state coincides with the ground state. Here it is intended to show that under specific conditions this quantum phase transition can manifest at finite temperature, although not as a symmetry breaking of a quantum state in pure form but as a change in the collective behavior of a grand canonical ensemble of particles.

\section{THERMODYNAMIC STATE OF THE OPEN SYSTEM}

When the system is embedded in a bath of inverse temperature $\beta$ and chemical potential $\mu$ it eventually reaches thermodynamic equilibrium. This equilibrium state is represented by a mixed state whose most important characterization is given by the grand canonical partition function

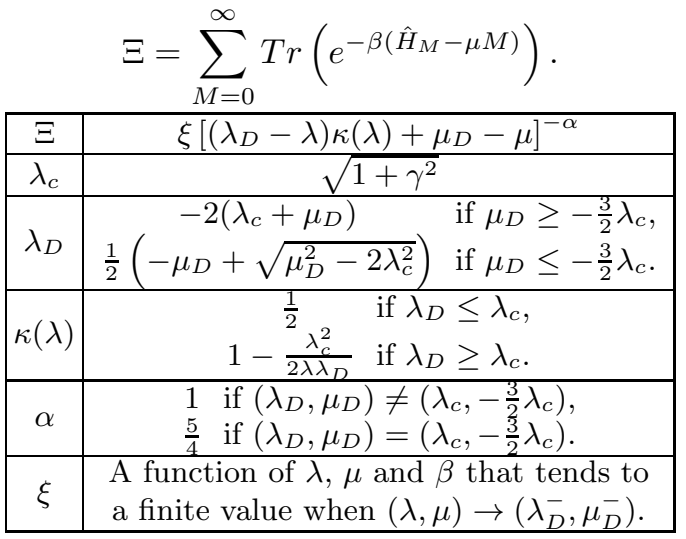

TABLE I: Grand partition function of Hamiltonian (1). Notice that $\lambda<\lambda_{D}$ and $\mu<\mu_{D}$.

The presence of non-quadratic terms in the Hamiltonian hinders the exact analytical calculation of $\Xi$. Hence, the following quadratic form is proposed

$$
\Xi \approx 1+\frac{1}{\sqrt{\pi}} \sum_{M=1}^{\infty} e^{\frac{\beta \lambda M}{2}} \int_{-\infty}^{\infty} d x e^{-x^{2}} \operatorname{Tr}\left(e^{-\beta\left(\hat{a}_{1}^{\dagger} \hat{a}_{2}+\hat{a}_{2}^{\dagger} \hat{a}_{1}\right)+i \beta \gamma\left(\hat{a}_{1}^{\dagger} \hat{a}_{2}-\hat{a}_{2}^{\dagger} \hat{a}_{1}\right)+x \sqrt{\frac{2 \beta \lambda}{M}}\left(\hat{m}_{1}-\hat{m}_{2}\right)+\beta \mu M}\right) .
$$

The original grand partition function can be obtained from this expression by carrying out the integral treating operators as scalars. This alternative expression is expected to be accurate over parameter zones where the system displays high occupation, since in this case fluctuations become negligible compared to mean values. As can be seen from appendix $B$, in the process of calculating (10) it is found that $\Xi$ converges inside the parameter zones highlighted in figure 1. The grand parti- tion function tends to diverge as any point of the curve $\left(\lambda_{D}, \mu_{D}\right)$, whose explicit expression is given in table प is approached from the left. Near this divergence, $\Xi$ displays the functionality reported also in table \. The line $\lambda=\lambda_{c}$ partitions the parameter space into cases whose properties are being discussed further ahead in this note. However, there is no symmetry breaking. In both cases the number of particles at both wells is the same. This is to be expected from the fact that the thermodynamic 

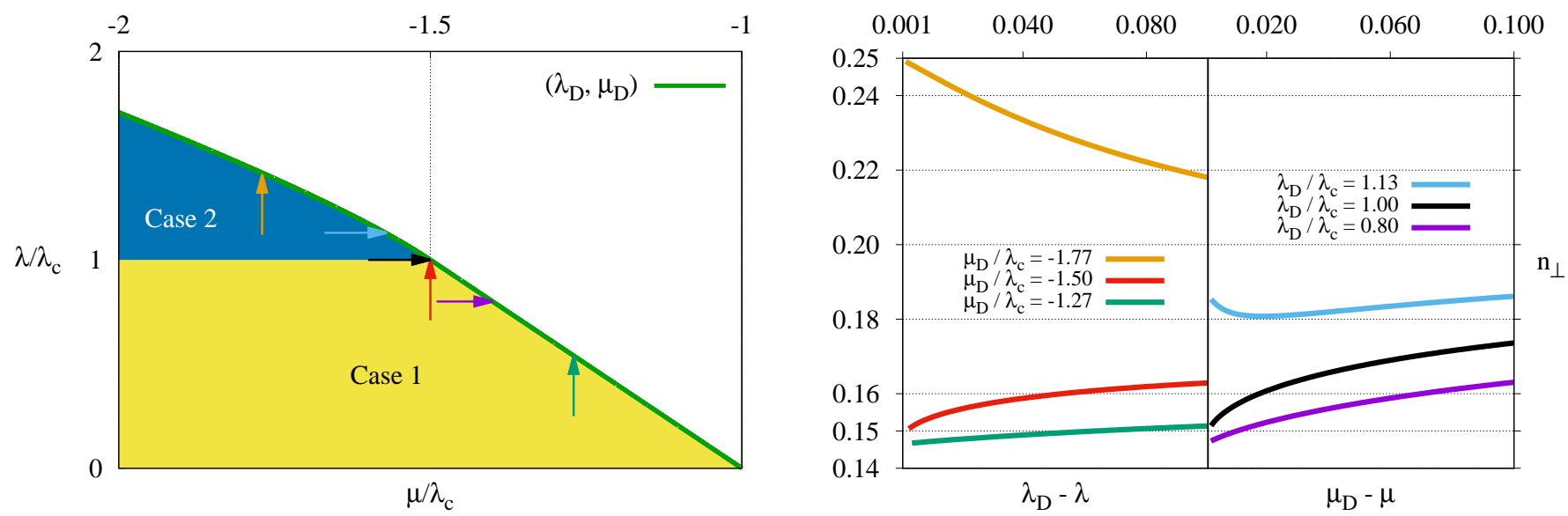

FIG. 1: Left. Colored regions represent the parameter zones where the grand partition function converged (equilibrium zones). Right. Relative mean number of particles in the mode perpendicular to the mode with maximum occupation, as defined by equation (20). Perpendicular occupation falls when approaching the divergence from inside the case- 1 region or through the boundary separating the cases, showing that to first order the particles tend to gather all in the mode with maximum occupation. This mode coincides with the ground state mode in the range $\lambda<\lambda_{c}$. The case is different when approaching the divergence from the case- 2 region, where the perpendicular occupation tends to a finite number. The values $\gamma=1$ and $\beta=1$ were taken to produce the graphs at the right panel.

\begin{tabular}{|c|c|c|c|}
\hline Observable & Formal expression & Leading term close to $\left(\lambda_{D}, \mu_{D}\right)$ & Behavior near $\left(\lambda_{D}, \mu_{D}\right)(\times \mathscr{M})$ \\
\hline $\mathscr{M}=\left\langle\hat{m}_{1}+\hat{m}_{2}\right\rangle$ & $\frac{1}{\beta} \frac{\partial \log \Xi}{\partial \mu}$ & $\frac{\alpha}{\beta}\left[\kappa\left(\lambda_{D}\right)\left(\lambda_{D}-\lambda\right)+\mu_{D}-\mu\right]^{-1}$ & 1 \\
\hline$E=\langle\hat{H}\rangle$ & $\mu \mathscr{M}-\frac{\partial \log \Xi}{\partial \beta}$ & $\mu_{D} \mathscr{M}$ & $\begin{array}{l}-\lambda_{c}-\frac{\lambda_{D}}{2} \quad \text { if } \lambda_{D} \leq \lambda_{c} \\
-\lambda_{D}-\frac{\lambda_{c}^{2}}{2 \lambda_{D}} \quad \text { if } \lambda_{D} \geq \lambda_{c}\end{array}$ \\
\hline$I=\left\langle\frac{\hat{m}_{1}^{2}+\hat{m}_{2}^{2}}{M}\right\rangle$ & $\frac{1}{\beta} \frac{\partial \log \Xi}{\partial \lambda}$ & $\kappa\left(\lambda_{D}\right) \mathscr{M}$ & $\begin{array}{cl}\frac{1}{2} & \text { if } \lambda_{D} \leq \lambda_{c} \\
1-\frac{\lambda_{c}^{2}}{2 \lambda_{D}^{2}} & \text { if } \lambda_{D} \geq \lambda_{c}\end{array}$ \\
\hline$J=-i\left\langle\hat{a}_{1}^{\dagger} \hat{a}_{2}-\hat{a}_{2}^{\dagger} \hat{a}_{1}\right\rangle$ & $-\frac{1}{\beta} \frac{\partial \log \Xi}{\partial \gamma}$ & $\frac{\gamma \kappa\left(\lambda_{D}\right)}{\lambda_{c}} \frac{\partial \lambda_{D}}{\partial \lambda_{c}} \mathscr{M}$ & $\begin{array}{ll}-\frac{\gamma}{\lambda_{c}} & \text { if } \lambda_{D} \leq \lambda_{c} \\
-\frac{\gamma}{\lambda_{D}} & \text { if } \lambda_{D} \geq \lambda_{c}\end{array}$ \\
\hline$W=\left\langle\hat{a}_{1}^{\dagger} \hat{a}_{2}+\hat{a}_{2}^{\dagger} \hat{a}_{1}\right\rangle$ & $E-\gamma J+\lambda I$ & {$\left[\mu_{D}+\kappa\left(\lambda_{D}\right)\left(\lambda_{D}-\frac{\gamma^{2}}{\lambda_{c}} \frac{\partial \lambda_{D}}{\partial \lambda_{c}}\right)\right] \mathscr{M}$} & $\begin{array}{ll}-\frac{1}{\lambda_{c}} & \text { if } \lambda_{D} \leq \lambda_{c} \\
-\frac{1}{\lambda_{D}} & \text { if } \lambda_{D} \geq \lambda_{c} .\end{array}$ \\
\hline
\end{tabular}

TABLE II: Mean values according to the grand canonical formalism. Unreferenced variables are identified in table [

state is a function of the Hamiltonian an as such it must display its symmetries. A number of observables can be obtained as derivatives of the grand partition function. These can be seen in table [I]. They all show the same scaling behavior near divergence, namely, proportional to the total number of particles. However, multiplicative coefficients display different functionality depending on the region of parameter space from which the divergence is approached. The relation between any pair of observables is continuous, but the relation between an observable and the divergence point presents a discontinuity at $\left(\lambda_{D}, \mu_{D}\right)=\left(\lambda_{c},-\frac{3}{2} \lambda_{c}\right)$. Thus, at finite temperature the parameter space is divided into two cases that can be recognized according to their divergence coefficients.

\section{POPULATION DISTRIBUTION}

Knowing that observables diverge when the system's parameters approach an established boundary in parameter space, it is of interest to uncover some of the features that characterize the thermodynamic state near this divergence zone. One way of addressing this issue is to find how much a given mode contributes to the thermodynamic state. Although bosons can access two independent modes, one for each well in Hamiltonian (1), it is possible to build other (linearly dependent) modes as superpositions of the original ones. In this scenario it becomes relevant to find the mean occupation of a given mode. For this purpose let us define the following weight function

$$
N(\theta, \varphi)=\left\langle\hat{b}^{\dagger} \hat{b}\right\rangle, \hat{b}^{\dagger}=\hat{a}_{1}^{\dagger} \cos \theta-\hat{a}_{2}^{\dagger} e^{i \varphi} \sin \theta .
$$


The average is calculated over the thermodynamic state associated with the grand partition function previously introduced in this document. Any mode available in physical space can be reached in the range $0 \leq \theta \leq \pi / 2$ and $0 \leq \varphi<2 \pi$. From a direct calculation it can be shown that this weight function can also be written as

$$
N=\frac{\mathscr{M}}{2}-\frac{\sin 2 \theta}{2}(W \cos \varphi+J \sin \varphi) .
$$

In the process of arriving at this expression the property $\left\langle\hat{m}_{1}-\hat{m}_{2}\right\rangle=0$ was used. This property follows from the fact that the equilibrium state must display the Hamiltonian's inversion symmetry. An explicit form of $N$ can be obtained by replacing the mean values of table $\prod_{\text {in }}$ (12). A pair of angles $\left(\theta^{\mathbf{\Lambda}}, \varphi^{\mathbf{\Lambda}}\right)$ defining a mode with maximum contribution must fulfill

$$
\begin{aligned}
\left.\partial_{\theta} N\right|_{\theta^{\mathbf{\Lambda}} \varphi^{\mathbf{\Lambda}}} & =-\cos 2 \theta^{\mathbf{\Lambda}}\left(W \cos \varphi^{\mathbf{\Lambda}}+J \sin \varphi^{\mathbf{\Lambda}}\right)=0 \\
\left.\partial_{\varphi} N\right|_{\theta^{\mathbf{\Lambda}} \varphi^{\mathbf{\Lambda}}} & =\frac{\sin 2 \theta^{\mathbf{\Lambda}}}{2}\left(W \sin \varphi^{\mathbf{\Lambda}}-J \cos \varphi^{\mathbf{\Lambda}}\right)=0 \\
\left.\partial_{\theta^{2}}^{2} N\right|_{\theta^{\mathbf{\Delta}} \varphi^{\mathbf{\Lambda}}} & =2 \sin 2 \theta^{\mathbf{\Lambda}}\left(W \cos \varphi^{\mathbf{\Lambda}}+J \sin \varphi^{\mathbf{\Lambda}}\right)<0 \\
& \left.4 \partial_{\varphi^{2}}^{2} N\right|_{\theta^{\mathbf{\Lambda}} \varphi^{\mathbf{\Lambda}}}=\left.\partial_{\theta^{2}}^{2} N\right|_{\theta^{\mathbf{\Lambda}} \varphi^{\mathbf{\Lambda}}}<0
\end{aligned}
$$

As can be seen, condition (15) implies (16), so it is really three conditions that must be accounted for. Equation (13) is met for $\theta^{\boldsymbol{\Lambda}}=\pi / 4$, giving a state with equal occupation at both sides, in compliance with the Hamiltonian's symmetry. According to table $\Pi$ both $W$ and $J$ are negative in the zone near divergence, hence conditions (14) and (15) are satisfied for

$$
\cos \varphi^{\mathbf{\Lambda}}=\frac{1}{\sqrt{1+t^{2}}}, \sin \varphi^{\mathbf{\Lambda}}=\frac{t}{\sqrt{1+t^{2}}}, t=\frac{J}{W}=\gamma .
$$

Interestingly, these parameters also define the ground state in the gaped phase $\left(\lambda<\lambda_{c}\right)$ of the quantum phase transition discussed in the first part of this work, but in this case this state with maximum occupation applies to the whole spectrum of parameters near divergence, including the gapless phase. The maximum occupation number is given by

$$
N^{\mathbf{\Lambda}}=N\left(\theta^{\mathbf{\Delta}}, \varphi^{\mathbf{\Delta}}\right)=\frac{\mathscr{M}}{2}+\frac{1}{2} \sqrt{W^{2}+J^{2}} .
$$

Inserting the values reported in table $\amalg$ it results

$$
n=\frac{N^{\boldsymbol{\Lambda}}}{\mathscr{M}}= \begin{cases}1 & \text { if } \lambda_{D} \leq \lambda_{c} \\ \frac{1}{2}\left(1+\frac{\lambda_{c}}{\lambda_{D}}\right) & \text { if } \lambda_{D} \geq \lambda_{c} .\end{cases}
$$

As below $\lambda_{c}$ the number of particles in the most populated mode coincides with the mean number of particles in the whole system, it is argued that in this regime particles go all into the maximally occupied mode, which is at the same time the ground state of the high-density Hamiltonian. The quantity

$$
n_{\perp}=1-n,
$$

is also the relative mean number of particles in the mode perpendicular to $\hat{b}^{\dagger}, \hat{d}^{\dagger}=\hat{a}_{1}^{\dagger} \cos \theta+\hat{a}_{2}^{\dagger} e^{i \varphi} \sin \theta$, evaluated at $\left(\theta^{\mathbf{\Delta}}, \varphi^{\mathbf{\Lambda}}\right)$. It then follows that for the case-1 family of parameters of figure1 the number of particles in the maximally occupied state approaches the mean number of particles in the system at the same time that the number of particles in the respective perpendicular state becomes negligible. Instead, in the case- 2 region the population is distributed over two modes. Take into account that this derivation is valid near the curve of divergence and not necessarily over the whole space of parameters.

A numerical study is undertaken in order to benchmark these analytical results. Hamiltonian (1) is diagonalized for various system sizes $M$ and the respective eigenvalues $E_{j}^{M}$ are used to find the grand canonical partition function thus

$$
\Xi=1+\sum_{M=1}^{M_{a x}} e^{\beta \mu M} \sum_{j=1}^{M+1} e^{-\beta E_{j}^{M}} .
$$

The maximum size $M_{a x}$ is adjusted depending on the system's parameters to achieve an absolute accuracy of $10^{-7}$. Eigenvalues can be in addition employed to calculate the mean number of particles $\mathscr{M}$ using a similar expression. Eigenvectors are also calculated and used to find $J$ and $W$ as weighed sums (not as numerical derivatives) in similarity to (21). These values are then used to find $n_{\perp}$. The results are shown in the right panel of figure 1. The numerical values of $n_{\perp}$ show a tendency that is consistent with the analytical study, displaying a monotonously decaying pattern as the divergence is approached by either $\lambda$ or $\mu$ from the case- 1 region. The same tendency is observed when the divergence is approached through the line that divides the parameter space. The decaying is stronger when approaching $\left(\lambda_{D}, \mu_{D}\right)=\left(\lambda_{c},-\frac{3}{2} \lambda_{c}\right)$ (black and red curves), scaling as $\approx x^{0.02}$ close to the origin. In other cases the decaying coefficient is smaller and seems to be dependent on the divergence point. Furthermore, $n_{\perp}$ grows when approaching $\lambda_{D}$ or $\mu_{D}$ at close range from the case- 2 region, suggesting a macroscopic fraction of particles settle in the perpendicular mode. These features show that the properties of the high-density ground state dominate the collective response in thermodynamic equilibrium [23]. The gaped phase transmutes into a collective state in which bosons cluster together in a single mode. This behavior seems to be independent of temperature, rather being determined by the proximity of interaction and chemical potential to the divergence region. Nevertheless, it is likely that temperature would define how fast collectivism arises as the divergence is approached. The fact that compresibility, which can be found as $\partial_{\mu} \mathscr{M}$, goes to infinity in general as $(\lambda, \mu) \rightarrow\left(\lambda_{D}, \mu_{D}\right)$ is indicative of a highly conductive state not necessarily correlated with collectivism.

A condensate is traditionally understood as the macroscopic occupation of a single-body state that in normal conditions displays only marginal occupation, like any 
other state in the spectrum. Here the situation is seen from the perspective that two occupation states are initially macroscopically occupied and then one of them is depleted under the right conditions. The end result is the same if the depleted state is seen as the set of marginally occupied states in the condensate.

\section{CONCLUSIONS}

The equilibrium response of a bosonic system displaying a quantum phase transition has been studied using the grand canonical formalism. The phases characterizing the zero-temperature state drive characteristic features in the finite temperature regime near the parameter zone where the partition function diverged. A form of quantum collectivism in which occupation is concentrated in the ground-state mode has been identified. Too much attraction tends to dissolve this state, but there exist a critical value of the interaction constant below which collectivism can prevail in the presence of attractive interaction. This is related to the effect that the negative chemical potential considered here has on balancing the instability caused by the attractive interaction and the fact that the interaction constant has been rescaled with respect to the system size. Moreover, according to the phase diagram in figure 1 there cannot be a condensed phase for positive values of the chemical potential. Perhaps the most critical aspect of the present study is therefore the consideration of negative chemical potential, which might pose technical challenges in a controlled scenario [24], but could take place spontaneously in a less-artificial one, as for example a bosonic superfluid. It remains to be seen whether other forms of collectivism can be induced by deliberately breaking the system's inversion symmetry, for instance, by setting different chemical-potential variables at each well. Also of interest is to scale up the model by considering a large number of modes in one dimension and see whether the collectivism observed in the small system can be a precursor of condensation in an infinite chain. Ultimately, by adding bosonic modes describing molecules it should be possible to study the interplay between attractiveness and molecular formation.

\section{References}

[1] F. Dalfovo, S. Giorgini, L. Pitaevskii and S. Stringari Theory of Bose-Einstein condensation in trapped gases Reviews of Modern Physics 71463 (1999).

[2] J. Kim, Y. Choi and E. Lee Collapse of the metastable state in an attractive Bose-Einstein condensate Physical Review E 66017201 (2002).

[3] G. Berman, A. Smerzi and A. Bishop Quantum Instability of a Bose-Einstein Condensate with Attractive Interaction Physical Review Letters 88120402 (2002).

[4] J. Bronski, L. Carr, R. Carretero-González, B. Deconinck, J. Kutz and K. Promislow Stability of attractive
Bose-Einstein condensates in a periodic potential Physical Review E 64056615 (2001).

[5] J. Gerton, D. Strekalov, I. Prodan and R. Hulet Direct observation of growth and collapse of a Bose-Einstein condensate with attractive interactions Nature 408692 (2000).

[6] L. Berge, T. Alexander and Y. Kivshar Stability criterion for attractive Bose-Einstein condensates Physical Review A 62023607 (2000).

[7] A. Eleftheriou and K. Huang Instability of a BoseEinstein condensate with an attractive interaction Physical Review A 61043601 (2000).

[8] M. Ueda and K. Huang Fate of a Bose-Einstein condensate with an attractive interaction Physical Review A 60 (1999).

[9] N. Wilkin, J. Gunn, and R. Smith Do attractive bosons condense? Physical Review Letters 802265 (1998).

[10] C. Sackett, H. Stoof and R. Hulet Growth and Collapse of a Bose-Einstein Condensate with Attractive Interactions Physical Review Letters 802031 (1998).

[11] H. Shi and W. Zheng Bose-Einstein condensation in an atomic gas with attractive interactions Physical Review A 552930 (1997).

[12] R. Dodd, M. Edwards, C. Williams, C. Clark, M. Holland, P. Ruprecht and K. Burnett Role of attractive interactions on Bose-Einstein condensation Physcal Review A 54661 (1996).

[13] C. Bradley, C. Sackett, J. Tollett and R. Hulet Evidence of Bose-Einstein Condensation in an Atomic Gas with Attractive Interactions Physical Review Letters 751687 (1995).

[14] F. Arute et al. Quantum supremacy using a programmable superconducting processor Nature $\mathbf{5 7 4} 505$ (2019).

[15] H. Zhong et al. Quantum computational advantage using photons Science 10.1126/science.abe8770 (2020).

[16] Z. Zhang, L. Chen, K. Yao and C. Chin Atomic BoseEinstein condensate to molecular Bose-Einstein condensate transition Nature 592708 (2021).

[17] C. Warner, A. Lam, N. Bigagli, H. Liu, I. Stevenson and S. Will Overlapping Bose-Einstein Condensates of ${ }^{23} \mathrm{Na}$ and ${ }^{133}$ Cs arXiv:2106.01334 (2021).

[18] R. Spekkens and J. Sipe Spatial fragmentation of a BoseEinstein condensate in a double-well potential Physical Review A 593868 (1999).

[19] M. Fisher, P. Weichman, G. Grinstein and D. Fisher Boson localization and the superfluid-insulator transition Physical Review B 40546 (1989).

[20] M. Greiner, O. Mandel, T. Esslinger, T. Hansch and I. Bloch Quantum phase transition from a superfluid to a Mott insulator in a gas of ultracold atoms Nature 41539 (2002).

[21] M. Aidelsburger, M. Atala, M. Lohse, J. Barreiro, B. Paredes and I. Bloch Realization of the Hofstadter Hamiltonian with Ultracold Atoms in Optical Lattices 111 185301 (2013).

[22] K. Jimenez and J. Reslen Thermodynamic signatures of an underlying quantum phase transition: A grand canonical approach Physics Letters A 3802603 (2016).

[23] K. Zhang and Z. Song Quantum phase transition in a quantum Ising chain at nonzero temperatures Physical Review Letters 126116401 (2021).

[24] T. Schaetz Trapping ions and atoms optically Journal of Physics B: Atomic, Molecular and Optical Physics 50 
102001 (2017).

\section{Appendix A: Quantum Phase Transition}

Making $\delta=1$ Hamiltonian (1) becomes

$$
\begin{gathered}
\hat{H}_{M}=\hat{a}_{1}^{\dagger} \hat{a}_{2}+\hat{a}_{2}^{\dagger} \hat{a}_{1}-i \gamma\left(\hat{a}_{1}^{\dagger} \hat{a}_{2}-\hat{a}_{2}^{\dagger} \hat{a}_{1}\right) \\
-\frac{\lambda}{M}\left(\hat{a}_{1}^{\dagger} \hat{a}_{1} \hat{a}_{1}^{\dagger} \hat{a}_{1}+\hat{a}_{2}^{\dagger} \hat{a}_{2} \hat{a}_{2}^{\dagger} \hat{a}_{2}\right) .
\end{gathered}
$$

It is assumed that the ground state can be written as

$$
\begin{gathered}
|G(\theta, \varphi)\rangle=\frac{\hat{b}_{1}^{\dagger}|0,0\rangle}{\sqrt{M !}}=|M, 0\rangle, \\
\hat{b}_{1}^{\dagger}=\hat{a}_{1}^{\dagger} \cos \theta-\hat{a}_{2}^{\dagger} e^{i \varphi} \sin \theta,
\end{gathered}
$$

so that $\left[\hat{b}_{1}, \hat{b}_{1}^{\dagger}\right]=1$. The angle domains are $0 \leq \theta \leq \frac{\pi}{2}$ and $0 \leq \varphi<2 \pi$, in such a way that different angle pairs correspond to different modes. The basis change is given by

$$
\begin{gathered}
{\left[\begin{array}{l}
\hat{b}_{1}^{\dagger} \\
\hat{b}_{2}^{\dagger}
\end{array}\right]=\left[\begin{array}{cc}
\cos \theta & -e^{i \varphi} \sin \theta \\
e^{-i \varphi} \sin \theta & \cos \theta
\end{array}\right]\left[\begin{array}{l}
\hat{a}_{1}^{\dagger} \\
\hat{a}_{2}^{\dagger}
\end{array}\right]} \\
\Rightarrow\left[\begin{array}{c}
\hat{a}_{1}^{\dagger} \\
\hat{a}_{2}^{\dagger}
\end{array}\right]=\left[\begin{array}{cc}
\cos \theta & e^{i \varphi} \sin \theta \\
-e^{-i \varphi} \sin \theta & \cos \theta
\end{array}\right]\left[\begin{array}{l}
\hat{b}_{1}^{\dagger} \\
\hat{b}_{2}^{\dagger}
\end{array}\right] .
\end{gathered}
$$

From direct calculations the following results are obtained

$$
\begin{gathered}
\left\langle G\left|\hat{a}_{1}^{\dagger} \hat{a}_{2}\right| G\right\rangle=-M e^{i \varphi} \cos \theta \sin \theta \\
\left\langle G\left|\hat{a}_{1}^{\dagger} \hat{a}_{1} \hat{a}_{1}^{\dagger} \hat{a}_{1}\right| G\right\rangle=M^{2} \cos ^{4} \theta+M \cos ^{2} \theta \sin ^{2} \theta \\
\left\langle G\left|\hat{a}_{2}^{\dagger} \hat{a}_{2} \hat{a}_{2}^{\dagger} \hat{a}_{2}\right| G\right\rangle=M^{2} \sin ^{4} \theta+M \cos ^{2} \theta \sin ^{2} \theta .
\end{gathered}
$$

Using these values the energy becomes to leading order in $M$

$$
\begin{gathered}
E_{G}(\theta, \varphi)=\left\langle G(\theta, \varphi) \mid \hat{H}_{M} G(\theta, \varphi)\right\rangle= \\
-M\left(\sin 2 \theta(\cos \varphi+\gamma \sin \varphi)+\lambda\left(1-\frac{\sin ^{2} 2 \theta}{2}\right)\right) .
\end{gathered}
$$

The ground state energy corresponds to the minimum of this expression with respect to $\theta$ and $\varphi$. Defining

$$
q(\theta, \varphi)=\sin 2 \theta(\cos \varphi+\gamma \sin \varphi)-\frac{\lambda \sin ^{2} 2 \theta}{2},
$$

extreme points must satisfy

$$
\begin{gathered}
\left.\partial_{\theta} q\right|_{\theta^{\star} \varphi^{\star}}=\cos 2 \theta^{\star}\left(\cos \varphi^{\star}+\gamma \sin \varphi^{\star}-\lambda \sin 2 \theta^{\star}\right)=0 \\
\left.\partial_{\varphi} q\right|_{\theta^{\star} \varphi^{\star}}=-\sin 2 \theta^{\star}\left(\sin \varphi^{\star}-\gamma \cos \varphi^{\star}\right)=0 \\
\begin{array}{c}
\left.\partial_{\theta^{2}}^{2} q\right|_{\theta^{\star} \varphi^{\star}}=-2 \sin 2 \theta^{\star}\left(\cos \varphi^{\star}+\gamma \sin \varphi^{\star}-\lambda \sin 2 \theta^{\star}\right) \\
-\lambda \cos ^{2} 2 \theta^{\star}<0 \\
\left.\partial_{\varphi^{2}}^{2} q\right|_{\theta^{\star} \varphi^{\star}}=\sin 2 \theta^{\star}\left(-\cos \varphi^{\star}-\gamma \sin \varphi^{\star}\right)<0
\end{array}
\end{gathered}
$$

Conditions (A11), are all met for the next values

$$
\theta^{\star}=\frac{\pi}{4}, \quad \cos \varphi^{\star}=\frac{1}{\sqrt{1+\gamma^{2}}}, \quad \sin \varphi^{\star}=\frac{\gamma}{\sqrt{1+\gamma^{2}}} .
$$

Condition (A13) is met for these same values in the range $\lambda<\sqrt{1+\gamma^{2}}$, which defines the scope of this particular physical phase. Replacing these extreme points in (A9) the ground state energy in this phase is found to be

$$
E_{G}=-M\left(\sqrt{1+\gamma^{2}}+\frac{\lambda}{2}\right) .
$$

Correspondingly, over the range $\lambda>\sqrt{1+\gamma^{2}}$ the following values constitute solutions

$$
\begin{gathered}
\theta_{1}^{\star}=\frac{1}{2} \arcsin \frac{\sqrt{1+\gamma^{2}}}{\lambda}, \theta_{2}^{\star}=\frac{\pi}{2}-\theta_{1}^{\star}, \\
\cos \varphi^{\star}=\frac{1}{\sqrt{1+\gamma^{2}}}, \quad \sin \varphi^{\star}=\frac{\gamma}{\sqrt{1+\gamma^{2}}} .
\end{gathered}
$$

The solutions are given by the pairs $\left\{\theta_{1}^{\star}, \varphi^{\star}\right\}$ and $\left\{\theta_{2}^{\star}, \varphi^{\star}\right\}$. Either pair delivers the following ground state energy

$$
E_{G}=-M\left(\lambda+\frac{1+\gamma^{2}}{2 \lambda}\right)
$$

The expression

$$
\lambda_{c}=\sqrt{1+\gamma^{2}}
$$

is the transition's critical point. Since these results correspond to first order in $M$, the quantum phase transition takes place in the high density limit, i.e., $M \rightarrow \infty$.

\section{Appendix B: Calculation of $\Xi$}

Taking equation (10) and making the variable change $x=y \sqrt{M \beta}$ leads to the next expression

$$
\Xi \approx 1+\sqrt{\frac{\beta}{\pi}} \sum_{M=1}^{\infty} \sqrt{M} e^{\frac{\beta \lambda M}{2}} \int_{-\infty}^{\infty} d y e^{-\beta M y^{2}} \operatorname{Tr}\left(e^{\beta \hat{h}_{M}}\right),
$$

so that

$$
\begin{gathered}
\hat{h}_{M}=-\left(\hat{a}_{1}^{\dagger} \hat{a}_{2}+\hat{a}_{2}^{\dagger} \hat{a}_{1}\right)+i \gamma\left(\hat{a}_{1}^{\dagger} \hat{a}_{2}-\hat{a}_{2}^{\dagger} \hat{a}_{1}\right) \\
+\sqrt{2 \lambda} y\left(\hat{m}_{1}-\hat{m}_{2}\right)+\mu\left(\hat{m}_{1}+\hat{m}_{2}\right) .
\end{gathered}
$$

This effective Hamiltonian can also be written as

$$
\hat{h}_{M}=\left(\begin{array}{ll}
\hat{a}_{1}^{\dagger} & \hat{a}_{2}^{\dagger}
\end{array}\right)\left(\begin{array}{cc}
\mu+\sqrt{2 \lambda} y & -1+i \gamma \\
-1-i \gamma & \mu-\sqrt{2 \lambda} y
\end{array}\right)\left(\begin{array}{c}
\hat{a}_{1} \\
\hat{a}_{2}
\end{array}\right) .
$$

Normal eigenenergies of $\hat{h}_{M}$ are then found to be

$$
\epsilon_{ \pm}(y)=\mu \pm \sqrt{2 \lambda y^{2}+1+\gamma^{2}}=\mu \pm \sqrt{2 \lambda y^{2}+\lambda_{c}^{2}} .
$$


Using these values the trace can be calculated as follows

$$
\begin{gathered}
\operatorname{Tr}\left(e^{\beta \hat{h}_{M}}\right)=\operatorname{Tr}\left(e^{\beta\left(\epsilon_{+} \hat{b}_{+}^{\dagger} \hat{b}_{+}+\epsilon_{-} \hat{b}_{-}^{\dagger} \hat{b}_{-}\right)}\right) \\
=\sum_{n=0}^{M} e^{\beta \epsilon_{+} n+\beta \epsilon_{-}(M-n)}=\frac{e^{\beta M \epsilon_{-}}-e^{\beta\left(\epsilon_{+}-\epsilon_{-}\right)} e^{\beta M \epsilon_{+}}}{1-e^{\beta\left(\epsilon_{+}-\epsilon_{-}\right)}} .
\end{gathered}
$$

Operators $\hat{b}_{+}$and $\hat{b}_{-}$correspond to diagonal bosonic modes. Now let us take a term from (B1) and reformulated in the next way

$$
\sqrt{M} e^{\frac{\beta \lambda M}{2}}=\frac{\sqrt{\beta} M}{\sqrt{\pi}} \int_{-\infty}^{\infty} d x e^{-\beta M x^{2}+\beta M \sqrt{2 \lambda} x} .
$$

Replacing (B5) and (B6) in (B1) and organizing terms yields

$$
\begin{gathered}
\Xi=1+\frac{\beta}{\pi} \int_{-\infty}^{\infty} d x \int_{-\infty}^{\infty} d y \frac{\sum_{M=1}^{\infty} M e^{\beta M\left(-x^{2}-y^{2}+\sqrt{2 \lambda} x+\epsilon_{+}(y)\right)}}{1-e^{-\beta\left(\epsilon_{+}(y)-\epsilon_{-}(y)\right)}} \\
+\frac{\sum_{M=1}^{\infty} M e^{\beta M\left(-x^{2}-y^{2}+\sqrt{2 \lambda} x+\epsilon_{-}(y)\right)}}{1-e^{\beta\left(\epsilon_{+}(y)-\epsilon_{-}(y)\right)}} .
\end{gathered}
$$

Defining

$$
F(x, y)=-x^{2}-y^{2}+\sqrt{2 \lambda} x+\sqrt{2 \lambda y^{2}+\lambda_{c}^{2}}+\mu,
$$

it can be seen that both sums in (B7) shall converge if $F(x, y)<0$ for any real value of $x$ and $y$. For this it is necessary that

$$
F\left(x^{\star}, y^{\star}\right)=F^{\star}<0,
$$

where $x^{\star}$ and $y^{\star}$ demark the location of the function's global maximum. Through a calculus analysis it can be shown that two main cases arise

1. case 1: $0 \leq \lambda<\min \left(-2\left(\mu+\lambda_{c}\right), \lambda_{c}\right)$.

The function's only maximum is located at $\left(x^{\star}=\right.$ $\left.\sqrt{\lambda / 2}, y^{\star}=0\right)$. The system's parameters are compatible with the condition

$$
F^{\star}=\frac{\lambda}{2}+\lambda_{c}+\mu=-\frac{\left(\lambda_{D}-\lambda\right)}{2}-\left(\mu_{D}-\mu\right)<0,
$$

being $\lambda_{D}$ and $\mu_{D}$ a pair of constants satisfying $\lambda_{D}=$ $-2\left(\lambda_{c}+\mu_{D}\right)$. Equation (B10) highlights the fact that

$$
\lim _{\lambda \rightarrow \lambda_{D}^{-}, \mu \rightarrow \mu_{D}^{-}} F^{\star}=0 .
$$

2. case 2: $\frac{\mu}{\lambda_{c}}<-\frac{3}{2}$ and $\lambda_{c} \leq \lambda<\frac{1}{2}\left(-\mu+\sqrt{\mu^{2}-2 \lambda_{c}^{2}}\right)$.

The function displays two maxima located at

$$
\left(x^{\star}=\sqrt{\frac{\lambda}{2}}, y^{\star}=\sqrt{\frac{\lambda^{2}-\lambda_{c}^{2}}{2 \lambda}}\right),
$$

and

$$
\left(x^{\star}=\sqrt{\frac{\lambda}{2}}, y^{\star}=-\sqrt{\frac{\lambda^{2}-\lambda_{c}^{2}}{2 \lambda}}\right) .
$$

The system's parameters are compatible with the condition

$$
\begin{gathered}
F^{\star}=\lambda+\frac{\lambda_{c}^{2}}{2 \lambda}+\mu \\
=-\left(\lambda_{D}-\lambda\right)\left(1-\frac{\lambda_{c}^{2}}{2 \lambda \lambda_{D}}\right)-\left(\mu_{D}-\mu\right)<0
\end{gathered}
$$

such that $\lambda_{D}=\frac{1}{2}\left(-\mu_{D}+\sqrt{\mu_{D}^{2}-2 \lambda_{c}^{2}}\right)$. As in the previous case, equation (B11) is satisfied. Figure 1 depicts the two cases in a parameter map. The divergence parameters, $\left(\lambda_{D}, \mu_{D}\right)$, are related as follows

$$
\lambda_{D}= \begin{cases}-2\left(\lambda_{c}+\mu_{D}\right) & \text { if } \mu_{D} \geq-\frac{3}{2} \lambda_{c} \\ \frac{1}{2}\left(-\mu_{D}+\sqrt{\mu_{D}^{2}-2 \lambda_{c}^{2}}\right) & \text { if } \mu_{D} \leq-\frac{3}{2} \lambda_{c} .\end{cases}
$$

Solving the sums in (B7) within the established spaces of convergence the following result is obtained

$$
\begin{gathered}
\Xi \approx 1+\frac{\beta}{4 \pi} \int_{-\infty}^{\infty} d x \int_{-\infty}^{\infty} d y \frac{\operatorname{csch}^{2}\left(\frac{\beta}{2} F(x, y)\right)}{1-e^{-\beta \sqrt{ }(y)}} \\
+\frac{\operatorname{csch}^{2}\left(\frac{\beta}{2} G(x, y)\right)}{1-e^{\beta \sqrt{ }(y)}},
\end{gathered}
$$

where

$$
G(x, y)=F(x, y)-\sqrt{ }(y), \sqrt{ }(y)=2 \sqrt{2 \lambda y^{2}+\lambda_{c}^{2}} .
$$

Close to divergence, only the part of the integral with $F(x, y)$ in (B16) goes to infinity. Since in such a case $F(x, y)$ gets close to zero, the following approximation becomes applicable

$$
\begin{array}{r}
\int_{-\infty}^{\infty} d x \int_{-\infty}^{\infty} d y \frac{\operatorname{csch}^{2}\left(\frac{\beta}{2} F(x, y)\right)}{1-e^{-\beta \sqrt{ }(y)}} \\
\approx \frac{4}{\beta^{2}} \int_{-\infty}^{\infty} d x \int_{-\infty}^{\infty} d y \frac{e^{\beta F(x, y)}}{F(x, y)^{2}\left(1-e^{-\beta \sqrt{ }(y)}\right)}
\end{array}
$$

Neither exponential in the integrand has a significant contribution to the scaling pattern of the gran-partition function. Therefore, the integral is further approximated by

$$
\frac{4 e^{\beta F^{\star}}}{\beta^{2}\left(1-e^{-\beta \sqrt{ }\left(y^{\star}\right)}\right)} \int_{-\infty}^{\infty} d x \int_{-\infty}^{\infty} d y \frac{1}{F(x, y)^{2}} .
$$

The integral above can be approximated close to the divergence zone, but for this it is necessary to differentiate a number of subcases. 
3. case 1: $0 \leq \lambda<\min \left(-2\left(\mu+\lambda_{c}\right)\right)$

a. $\quad$ subcase 1: excluding $\left(\lambda_{D}, \mu_{D}\right)=\left(\lambda_{c},-\frac{3 \lambda_{c}}{2}\right)$

Function $F(x, y)$ is expanded and the first nonvanishing terms are retained. The resulting integral can be solved by standard methods, along the lines of

$$
\begin{gathered}
\int_{-\infty}^{\infty} d x \int_{-\infty}^{\infty} d y \frac{1}{F(x, y)^{2}} \approx \\
\int_{-\infty}^{\infty} d x \int_{-\infty}^{\infty} d y \frac{1}{\left(F^{\star}-\alpha_{x}^{2}\left(x-x^{\star}\right)^{2}-\alpha_{y}^{2}\left(y-y^{\star}\right)^{2}\right)^{2}} \\
=-\frac{\pi}{\left|\alpha_{x}\right|\left|\alpha_{y}\right| F^{\star}}
\end{gathered}
$$

being

$$
\alpha_{x}^{2}=-\left.\frac{1}{2} \frac{\partial^{2} F}{\partial x^{2}}\right|_{x=x^{\star}, y=y^{\star}}, \alpha_{y}^{2}=-\left.\frac{1}{2} \frac{\partial^{2} F}{\partial y^{2}}\right|_{x=x^{\star}, y=y^{\star}} .
$$

An approximated expression to the grand partition function can be obtained by gathering all the coefficients involved in the derivation. However, it is only the denominator in the last term of (B20) that determines the divergence trend. The resulting expression is valid inside the parameter zone corresponding to this case. Replacing the following identities in (B20):

$$
\begin{gathered}
F^{\star}=-\frac{\left(\lambda_{D}-\lambda\right)}{2}-\left(\mu_{D}-\mu\right), \\
\alpha_{x}^{2}=1, \\
\alpha_{y}^{2}=1-\frac{\lambda}{\lambda_{c}}
\end{gathered}
$$

the grand-partition function can be written as

$$
\Xi=\frac{\xi\left(\lambda, \mu, \lambda_{c}\right)}{\frac{\lambda_{D}-\lambda}{2}+\mu_{D}-\mu}
$$

Function $\xi\left(\lambda, \mu, \lambda_{c}\right)$ must be well behaved and continuous at the point $(\lambda, \mu)=\left(\lambda_{D}, \mu_{D}\right)$. The reason why this derivation cannot accommodate $\lambda_{D}=\lambda_{c}$ is because this would allow $\lambda$ to get infinitesimally close to $\lambda_{c}$, causing the vanishing of $\alpha_{y}$ in (B24).

b. $\quad$ subcase 2: $\left(\lambda_{D}, \mu_{D}\right)=\left(\lambda_{c},-\frac{3 \lambda_{c}}{2}\right)$.

As one of the second-order expansion-terms of $F(x, y)$ vanishes when $\lambda$ approaches $\lambda_{c}$, the next non-vanishing term of the expansion is considered in (B20). The resulting expression reads

$$
\begin{aligned}
& \int_{-\infty}^{\infty} d x \int_{-\infty}^{\infty} d y \frac{1}{\left(F^{\star}-\alpha_{x}^{2}\left(x-x^{\star}\right)^{2}-\gamma_{y}^{2}\left(y-y^{\star}\right)^{4}\right)^{2}}= \\
& \frac{1}{\left|\alpha_{x}\right| \sqrt{\left|\gamma_{y}\right|}\left(-F^{\star}\right)^{\frac{5}{4}}} \int_{0}^{\pi} \frac{d \theta}{\sqrt{\sin \theta}} \int_{0}^{\infty} d t \frac{\sqrt{t}}{\left(1+t^{2}\right)^{2}} .
\end{aligned}
$$

Replacing

$$
\begin{gathered}
F^{\star}=-\frac{\left(\lambda_{c}-\lambda\right)}{2}-\left(-\frac{3 \lambda_{c}}{2}-\mu\right), \\
\alpha_{x}^{2}=1 \\
\gamma_{y}^{2}=\frac{\lambda^{2}}{2 \lambda_{c}^{3}},
\end{gathered}
$$

As a consequence, the grand partition function adopts the next form

$$
\Xi=\frac{\xi\left(\lambda, \mu, \lambda_{c}\right)}{\left(\frac{\lambda_{c}-\lambda}{2}-\frac{3 \lambda_{c}}{2}-\mu\right)^{\frac{5}{4}}},
$$

in such a way that $\xi\left(\lambda, \mu, \lambda_{c}\right)$ be well behaved and continuous at $(\lambda, \mu)=\left(\lambda_{c},-\frac{3 \lambda_{c}}{2}\right)$.

4. case 2: $\frac{\mu}{\lambda_{c}}<-\frac{3}{2}$ and $\lambda_{c} \leq \lambda<\frac{1}{2}\left(-\mu+\sqrt{\mu^{2}-2 \lambda_{c}^{2}}\right)$.

a. $\quad$ subcase 3: excluding $\left(\lambda_{D}, \mu_{D}\right)=\left(\lambda_{c},-\frac{3 \lambda_{c}}{2}\right)$.

In this case function $F(x, y)$ has two maxima located at opposite sides of the $y$ axis. Formally, this would require to consider the contribution of two expansions, deriving in two integrals, each of which limited to half the plane. However, due to the symmetry of $F(x, y)$, this is effectively equivalent to considering twice one expansion integrated over the whole plane, since only the contribution around the expansion point is relevant. Proceeding in this way, the same expression (B20) found before is obtained. Likewise, replacing

$$
\begin{gathered}
F^{\star}=-\left(\lambda_{D}-\lambda\right)\left(1-\frac{\lambda_{c}^{2}}{2 \lambda_{D}^{2}}\right)-\left(\mu_{D}-\mu\right), \\
\alpha_{x}^{2}=1 \\
\alpha_{y}^{2}=1-\left(\frac{\lambda_{c}}{\lambda}\right)^{2},
\end{gathered}
$$

it follows that the grand partition function can be written as

$$
\Xi=\frac{\xi\left(\lambda, \mu, \lambda_{c}\right)}{\left(\lambda_{D}-\lambda\right)\left(1-\frac{\lambda_{c}^{2}}{2 \lambda \lambda_{D}}\right)+\left(\mu_{D}-\mu\right)}
$$

being $\xi\left(\lambda, \mu, \lambda_{c}\right)$ a well behaved and continuous function at $(\lambda, \mu)=\left(\lambda_{D}, \mu_{D}\right)$.

b. $\quad$ subcase $4:\left(\lambda_{D}, \mu_{D}\right)=\left(\lambda_{c},-\frac{3 \lambda_{c}}{2}\right)$.

In close parallel to subcase 2, function $F(x, y)$ is expanded and the first non-vanishing terms that survive in the limit $\lambda \rightarrow \lambda_{c}$ are retained. The resulting expression 
coincides with (B26). Replacing

$$
\begin{gathered}
F^{\star}=-\frac{\lambda_{c}-\lambda}{2}-\left(-\frac{3 \lambda_{c}}{2}-\mu\right), \\
\alpha_{x}^{2}=1, \\
\gamma_{y}^{2}=\frac{\lambda_{c}^{2}}{2 \lambda}\left(\lambda_{c}^{2}-4\left(\lambda^{2}-\lambda_{c}^{2}\right)\right),
\end{gathered}
$$

the final form of the grand-partition function is found to be analogous to (B30). The grand partition function can be written in a general way as

$$
\Xi=\frac{\xi}{\left[\left(\lambda_{D}-\lambda\right) \kappa(\lambda)+\mu_{D}-\mu\right]^{\alpha}} .
$$

Function $\xi$ does not contribute to the scaling pattern of $\Xi$ and makes no significant contribution to observables in the parameter zone near divergence. The other variables are given by

$$
\kappa(\lambda)= \begin{cases}\frac{1}{2} & \text { if } \lambda_{D} \leq \lambda_{c} \\ 1-\frac{\lambda_{c}^{2}}{2 \lambda \lambda_{D}} & \text { if } \lambda_{D} \geq \lambda_{c}\end{cases}
$$

and

$$
\alpha= \begin{cases}1 & \text { if }\left(\lambda_{D}, \mu_{D}\right) \neq\left(\lambda_{c},-\frac{3}{2} \lambda_{c}\right), \\ \frac{5}{4} & \text { if }\left(\lambda_{D}, \mu_{D}\right)=\left(\lambda_{c},-\frac{3}{2} \lambda_{c}\right) .\end{cases}
$$

